\title{
Regional Cooperation: The Case in Astronomy
}

\author{
Bambang Hidayat \\ Bosscha Observatory, Bandung Institute of Technology, Lembang 40391, \\ Indonesia
}

\section{Introduction}

In the Asia Pacific environment some astronomical centres already exist. It must be understood that while quite a few are already deeply involved in frontline astronomical research; others are still struggling to make their existence appreciated in their home countries, or, are trying to be recognized within the astronomical community. Whatever their status now, the basic underlying principle for the establishment of astronomy centres is the same and is motivated by the same ideals. These are to pursue scientific truths about the sun, planets, stars and galaxies.

It is often the case that inherent national problems (economic or political) can inadvertently push the astronomy institutions behind. Furthermore, there exists among the political elite in a country different, not to say lack of, visions and perceptions about science in general and astronomy in particular. In such a situation the scientists and astronomers in that country find themselves in a twofront battle-line. Firstly, they must try to overcome the shortages of manpower and funds for hardware, so that their institutions can grow. Secondly, they must elevate their national programmes so that they can be accepted by international research standards.

However, efforts grow in a setting determined by international requirements for astronomy. In this type of scenario, ambitions, goals and requirements also expand. These may be difficult to cope with, given meager means. Over a long period, a disparity between the fortunate and the less fortunate groups of astronomers can develop, thus putting the unfortunate further back.

Outside stimuli are needed in order to break the isolation and to ensure more meaningful scientific output. While this condition holds true in general, one should not fall into the trap of egalitarian principle in which external stimuli are simply viewed, or interpreted, as a means of distributing scientific wealth. Rather, such stimuli should be regarded as a means of creating seeds for scientific collaboration in the foreseeable future.

In the following paragraphs, some views on cooperation in astronomy are outlined, in which the aims are to promote self-reliance of the participating countries in the field of astronomy.

\section{Endogenous Capability}

In general, cooperation implies that genuine benefit should be obtained and distributed among the concerned parties. "Just-return" may be the right term in the economy of trade, but in the case of scientific collaboration this term 
should be interpreted. The concept of a just-return should not be confined to material benefit alone, but should accommodate a wider sense of understanding that collaboration which will serve as endowment for science on a longer timescale.

In the words of Salam (1993), whose exemplary efforts for third-world science have been recognized beyond any doubt, endowment in science for the future can best be secured by exposing scientists and institutions from the developing world to new trends and techniques in science. The same thing is true for astronomy. Networks of individuals and institutions who share common ideals should receive attention and helping hands so that their astronomical endeavours do not fall back into the drain.

Cooperation should also be able to provide, in time, an added value to other national undertakings, namely to increase the endogenous capability. Many potential scientific workers can be deprived from making contributions to modern scientific endeavour simply because the opportunities are not there. A prevailing absence of literature, for example, would produce detrimental effects for an establishment. When a scientist's aspirations become too lofty to be realized, the result is more often apathy than anything else, and there will be no chance of handing over scientific leadership. Salam (1993) also remarked "It should be noted that although progress in science may be achieved through breakthrough by towering individuals, advances in research are also due to collaborative work by teams which may be made up of as many as dozens or even hundreds of scientists". The author agrees with this notion which is particularly true for the developing countries and for the institutions which are on the brink of reaching a critical mass.

Looking at the problem from the point of view of the less fortunate groups of astronomers, the writer can ascertain that the pressing issue is the critical mass. Many countries understand this point but other social programmes unfortunately force the country to adopt measures which are not to the astronomers' advantage. It is imperative that cooperative projects should include scientific manpower programmes to ensure the continuation of scientific knowledge.

In many cases, astronomy is given a low priority rank, so efforts to improve manpower or hardware capabilities are hampered. It is clear that there is a great need to address a broad range of social goals, focusing more attention on human and social concerns. This does not mean that the technical content of science and astronomy should be put aside. As already stated, some astronomy centres already exist. With growing awareness and rapidly increasing globalization, it is not surprising that the need for more astronomy institutions is imminent. It is important to improve on hardware capability and to reach the critical number of astronomers for an institution. Such infra-structure can possibly be solved by bilateral agreements. It is then critically important to devote more attention to the long-term considerations of science and technology policies in order to bring the participating countries and institutions to a level at which they could benefit from such agreements.

If the creation of competitive advantages is understandably listed in the long-term agenda, astronomers might wonder whether there are items which can be included in shorter term planning. Many countries are well able to offer competitive services. Good sites for astronomical observations may be avail- 
able and telescopes of moderate sizes can be built on such sites. Naturally, the geographic distribution of telescopes is a factor which should be considered, in particular so that objects with time-varying phenomena can be monitored continuously. The author believes that this type of cooperation should be scrutinized in developing regional cooperation. This would allow not only division of labour among institutions but would also create self-reliance which is a very important ingredient in the future life of an institution.

The IAU has untiringly tried to make our astronomy community grow stronger. Some international organizations, UNESCO and ICTP to name two, have also put in their agenda programmes for developing basic science including astronomy. It is now up to us to make tighter working arrangements. There are also many bilateral agreements, such as the JSPS for Japan and other Asian countries and the Leids-Kerkhoven-Bosscha Foundation in the case of the Netherlands and Indonesia. We should look too with gratification at the efforts of the UN's commision on outer space which may provide funding for astronomy.

It is commonly perceived that spin-offs and the applicability of scientific endeavour form the primary factors in determining which projects should become a national priority. Astronomical cooperation should not be free from such judgement. However, astronomy has shown that it requires a high level of technology and sophisticated instrumentation. Spin-offs therefore can be expected from the introduction of new technology. Above all, it should not be forgotten that the transfer of technology should be accompanied by the transfer of science. For many countries, such scientific changes also lead to cultural changes. This is an important aspect of social-cultural relationships, although one has to realize that such effects are not easy to measure.

Thus, cooperation should aim at developing astronomy whilst at the same time should regard the needs of society for technological spin-offs (e.g Rhodes 1991).

\section{Cooperation: What Can Be Done}

One may ask - what can be done for developing nations in the Asia-Pacific region? This region includes many astronomy centres with various levels of economic capability and with a plurality of cultures. Because of these factors, extra efforts must be exerted so that both the fortunate and less fortunate institutions may share the common goals and concerns of astronomy.

As has been mentioned by Ishida (these proceedings), bilateral cooperation can be started on the basis of a common goal, and approached using comparative advantages such as geographical sites. Many young astronomers would be pleased to be allowed to use the most advanced instrumentation for their studies. However, limitations in telescope time and budgets may create obstacles in realizing such dreams. Some people may not be patient enough to start with smaller astronomical projects even though the projects deserve scientific merit. There are still a plethora of problems which can be undertaken with a rather small instrument, not only to satisfy the curiosity but also to add to the treasure of knowledge. Small as they may be, if done with devotion there will be fruits to be grasped. 
For general problems, institutions located at strategically located points can cooperate and combine their resources to:

1. observe objects which show transient and time phenomena.

2. search and survey to discover certain type of objects.

Using data handling and modern distribution methods (Isobe, these proceedings), the efforts can produce fruitful cooperation between countries. Results in the form of light curves of phenomena and the characterization of the nature of the objects are still welcome. This meeting has been concerned in particular with how to employ small Schmidt telescopes. While many large Schmidt telescopes are increasingly used for extragalactic researches, there are still galactic problems within the reach of smaller telescopes. These include:

1. Galactic structure studies

(a) study and characterization of planetary nebulae in relation to IRAS data

(b) the structure and kinematics of small $\mathrm{H}$ II regions

(c) star counts in relation with dark nebulae to obtain distances and other physical characteristics

(d) study of star forming regions in Bok globules and giant molecular clouds

(e) ascertain the nature of star groupings, in relation to the theory of cluster dispersal

2. variable-star surveys

3. near-space statistics of fast-moving-objects which include the study of near-earth satellites as well as imaging of comets.

These projects can be elaborated and are within the grasp of many institutions within the Asia-Pacific region.

\section{Conclusions}

- It should be borne in mind that the transfer of technology also means the transfer of science and culture. This, in turn, will help to create competitive advantages.

- To fill scientific gaps between countries any cooperative efforts should promote scientific manpower developments.

- While technical cooperation is an important aspect, one should produce endowment for the future of astronomy to provide for sustainable development.

- Many astronomical problems can be solved with regional cooperative efforts. They have scientific merits, in the sense that the results can add to the existing treasure of knowledge. 


\section{Acknowledgements}

The writer thanks Hakim L. Malasan for his help in finalizing this text and for valuable discussion.

\section{References}

Hidayat B., 1993, Proc. ISY Congress, 2, 52

Ishida K., 1994, these proceedings

Isobe $S ., 1994$, these proceedings

McCrea W. H., 1987, ARA\&A, 25, 1

Rhodes F. H. T., 1991, Physics Today, 42, 41

Salam A., 1993, in World Science Report, Anonym, (UNESCO, Paris), p. 166 